\title{
Brinkman volume penalization for bathymetry in three-dimensional ocean models
}

\author{
L. Debreu ${ }^{\mathrm{a}}$, N.K.-R. Kevlahan ${ }^{\mathrm{b}, *}$ \\ ${ }^{a}$ Univ. Grenoble Alpes, Inria, CNRS, Grenoble INP, LJK, 38000 Grenoble, France \\ ${ }^{b}$ Department of Mathematics and Statistics, McMaster University, Hamilton, Canada
}

\begin{abstract}
Accurate and stable implementation of bathymetry boundary conditions remains a challenging problem. The dynamics of ocean flow often depend sensitively on satisfying bathymetry boundary conditions and correctly representing their complex geometry. Generalized (e.g. $\sigma$ ) terrain-following coordinates are often used in ocean models, but they require smoothing the bathymetry to reduce pressure gradient errors (Mellor et al., 1994). Geopotential z-coordinates are a common alternative that avoid pressure gradient and numerical diapycnal diffusion errors, but they generate spurious flow due to their "staircase" geometry. We introduce a new Brinkman volume penalization to approximate the no-slip boundary condition and complex geometry of bathymetry in ocean models. This approach corrects the staircase effect of $z$-coordinates, does not introduce any new stability constraints on the geometry of the bathymetry and is easy to implement in an existing ocean model. The porosity parameter allows modelling subgrid scale details of the geometry. We illustrate the penalization and confirm its accuracy by applying it to
\end{abstract}

\footnotetext{
${ }^{*}$ Corresponding author.

Email addresses: Laurent.Debreu@inria.fr (L. Debreu), kevlahan@mcmaster.ca (N.K.-R. Kevlahan)
} 
two standard test flows: upwelling over a sloping bottom and internal tides over highly peaked bathymetry features. In future work we will explore applying the penalization to more realistic bathymetry configurations, and moving boundaries such as melting/freezing ice shelves.

Keywords: algorithms, bathymetry, bottom topography, computation, ocean modelling, penalization

\section{Introduction}

Penalization is a well-established way to implicitly enforce boundary conditions for complicated or moving geometries (e.g Peskin, 1972; Angot et al., 1999; Kevlahan and Ghidaglia, 2001). In such cases, explicitly imposing boundary conditions is computational expensive, inaccurate or requires making assumptions about geometrical properties of the boundary (e.g. smoothness, normal direction). In contrast, penalization methods are simple to implement since they typically only require adding additional source terms to the dynamical equations. The accuracy of the boundary conditions is controlled easily by modifying the values of one or more control parameters.

There are two main classes of penalization methods used in computational fluid dynamics: the immersed boundary method and volume penalization. In the first case, the boundary is represented as a collection of one dimensional fibres where the physics of the fibre is modelled using spring forces. These spring forces exert a force on the fluid and impose the desired boundary condition, and also model the mechanical properties of the solid. This method is appropriate when it is im- 
portant to represent the mechanics of the solid material, as in modelling a beating heart (Peskin, 1972).

We propose using the second approach, volume penalization, based on modelling the the solid as a porous medium. This method is well-motivated for ocean modelling since the mud and sand boundaries of the ocean are naturally porous. In addition, it is not clear that modelling coastlines and bathymetry as impermeable smooth curves is more physically realistic than using a method that requires no assumptions on the differentiability of the boundary. Volume penalization also works well for moving boundaries and fluid-structure interaction (e.g. Ghasemi and Kevlahan, 2017; Kevlahan, 2011, 2007)

Adcroft (2013) also proposed a form of volume penalization for subgrid scale of modelling of bathymetry that involved volume penalization. His goal was to capture the qualitative and quantitative features of small scale bathymetry on a coarser grid. To do this he represents each computational cell int terms of three layers of variable depth: an impermeable blocking layer, a permeable layer and a clear flow layer. The impermeable layer represents solid features in the subgrid scale bathymetry that block all flow, while the porosity of the permeable layer is is calculated based on the (non-blocking) subgrid scale details of the bathymetry. The blocking effect is represented by thin walls. The goal of this approach is to "homogenize" the subgrid scale features and retain essential blocking effects, rather than impose boundary conditions using a rigorous limit of porous medium flow as we propose here. In particular, Adcroft (2013) does not include a source 
term in the momentum equation to model the permeability of solid regions. We do, however, do some subgrid scale modelling to determine the porosity of cells near the boundary, which is similar to what Adcroft (2013) does in his porous layer.

In a series of papers (Lundquist et al., 2010, 2012; Bao et al., 2018) Lundquist and collaborators propose, implement and validate an immersed boundary method for flow over complex terrain in the weather research and forecasting (WRF) atmosphere model. The use of penalization was motivated by the observation that "With increased resolution, resolved terrain slopes become steeper, and the native terrain-following coordinates used in WRF result in numerical errors and instability." Ocean models using generalized coordinate suffer from similar instability and pressure gradient errors, which also motivates our penalization method. In contrast to the volume penalization approach we propose here, Lundquist et al. (2010)'s method uses surface penalization and cut cells and is not based on taking the limit of a physical model. However, their work has shown the potential of penalization to provide real advantages in accuracy and stability for geophysical models compared with explicitly implementing boundary conditions for complex topography. In common with Lundquist et al. (2010), we also use penalization to add details to a smooth coarse scale terrain following (generalized) coordinate system.

Reckinger et al. (2012) proposed a Brinkman volume penalization method for ocean modelling. However, it has fundamental differences compared with Kevlahan et al. (2015)'s approach, described below, because it is not derived formally from the shallow water equations in a porous medium. First, Reckinger et al. 
(2012)'s model includes porosity (non-conservatively) only in the mass conservation equation. This means that mass is not conserved. Secondly, their method does not ensure that mass and energy travel at the same speed (so energy is not conserved). Thirdly, the linear wave speed is much faster in the solid regions, which means their penalization scheme severely limits the time step and numerical stability of the numerical scheme. In addition, it appears that porosity is not used at all in the formulation of the three-dimensional hydrostatic version of their penalization method, which means there is only one parameter available to control the error.

Kevlahan et al. (2015) introduced a Brinkman penalization method for no-slip lateral boundary conditions in the two-dimensional shallow water equations based on flow in a porous medium characterized by its porosity $\phi(x)$ and permeability $\sigma(x)$. As in all penalization methods, the boundary conditions are implemented implicitly by modifying the equations. No-slip boundary conditions are then recovered approximately by setting the porosity and permeability to very small values in the solid region (the fluid regions are characterized by $\phi(x)=1$ and $\sigma(x)=0$ ). In the penalized system the prognostic variables are the height $\tilde{h}(x, t)=\phi(x) h(x, t)$ (proportional to the mass density) and horizontal velocity $\vec{u}(x, t)$.

The resulting penalized shallow equations are

$$
\begin{aligned}
\frac{\partial \tilde{h}}{\partial t}+\operatorname{div} \tilde{h} \vec{u} & =0, \\
\frac{\partial \vec{u}}{\partial t}+\frac{\operatorname{curl} \vec{u}}{\tilde{h}} \times \tilde{h} \vec{u}+\operatorname{grad}\left(\frac{g \tilde{\eta}}{\phi(x)}+\frac{1}{2}|\vec{u}|^{2}\right) & =-\sigma(x) \vec{u},
\end{aligned}
$$

where $x \in \mathbb{R}^{2}, \tilde{\eta}=\phi(x) \eta$ and $\eta$ is the perturbation the free surface, $h(x, t)=$ 
$H+\eta(x, t)$. The porosity $\phi(x)$ and permeability $\sigma(x)$ are discontinuous,

$$
(\phi(x), \sigma(x))=\left\{\begin{array}{cl}
(\alpha, 1 / \epsilon) & \text { in the penalized region, } \\
(1,0) & \text { in the fluid, }
\end{array}\right.
$$

with $\epsilon \ll \alpha \ll 1$, where $\alpha$ and $\epsilon$ are, respectively, the porosity and permeability parameters of the solid (porous) regions. According to Darcy's law, they are related according to $\epsilon=k /(\mu \alpha)$, where $k$ is the permeability of the solid and $\mu$ is the viscosity of the fluid. However, for the purposes of penalization, we will assume that $\alpha$ and $\epsilon$ can be varied independently. The solid regions are defined by the indicator function $\mathbb{1}(x)$,

$$
\mathbb{1}(x)=\left\{\begin{array}{cc}
1 & \text { in the solid, } \\
0 & \text { in the fluid. }
\end{array}\right.
$$

When implemented numerically the indicator function $\mathbb{1}(x)$ is smoothed over a few grid points, as discussed in Reckinger et al. (2012). This makes it easy to implement even very complex geometries since information about the geometry of boundary (e.g. local tangent and normal directions) are not needed and the computational grid need not conform to the boundary. The porosity $\phi(x)$ and permeability $\sigma(x)$ are then defined based on $\mathbb{1}(x)$ and the control parameters $\alpha \ll 1$ and $\epsilon \ll 1$ as

$$
\begin{aligned}
& \phi(x)=1+\mathbb{1}(x)(\alpha-1), \\
& \sigma(x)=\frac{1}{\epsilon} \mathbb{1}(x) .
\end{aligned}
$$

The penalization conserves mass and is stable (total energy is decreasing) and does not lead to higher wave speeds in the solid region. The error of the penaliza- 
tion is $O\left(\alpha \epsilon^{1 / 2}\right)$ and therefore the desired accuracy in the boundary conditions can be controlled by appropriately choosing the two parameters $\alpha$ and $\epsilon$. (Note that if we had used the physical definition of $\epsilon$ as $k /(\mu \alpha)$ then the error would be $O(\sqrt{\alpha \epsilon}$.)

In practice, $\epsilon$ is chosen so as not to limit the time step, e.g. $\epsilon \geq C \Delta t$ for an explicit method in time, and then $\alpha$ is set to achieve the desired error. Although the equations are solved in the entire computational domain, both fluid and solid, it is only necessary to compute the solution accurately in the solid region in a narrow "skin depth" of size $\delta$ inside the solid. In the inertia-gravity wave regime $\delta=$ $O(\sqrt{c L \epsilon})$, where $c$ is the barotropic wave speed and $L$ is a characteristic horizontal length scale. In the quasi-geostrophic regime $\delta=O(\sqrt{v \epsilon})$ or, more precisely, $\delta=5 \sqrt{v \epsilon}$ where $v$ is the viscosity. This suggests that the spatial resolution should satisfy $\delta / \Delta x \geq 2$ to properly resolve the skin depth. (Since Kevlahan et al. (2015) considered a dynamically adaptive method, very little computation was required in the solid region.)

The goal of this paper is to extend the Brinkman volume penalization method to three-dimensional primitive equations (hydrostatic, Boussinesq) ocean models in order to avoid some of the drawbacks of the usual vertical coordinate systems. In particular, we eliminate the "stair-case" effect associated with geopotential (or $z$-) coordinates and avoid the pressure gradient constraints of terrain-following $\sigma$ (or S-) generalized coordinates associated with steep (small scale) bathymetry features. The stair-case effect is due to the fact that geopotential layers will in general intersect with sloping bathymetry which leads to inaccurate representation of bot- 
tom currents (the stair-case can even generate its own gravity currents in some cases!). A pure terrain-following generalized coordinate suffers from inaccuracy in representing the horizontal pressure gradient and also may lead to artificial diapycnal diffusion Marchesiello et al. (2009) .

We combine aspects of both terrain-following and geopotential generalized coordinates to take advantage of the strengths of each system and minimize their weaknesses.

First, a terrain-following (i.e. $\sigma$ ) coordinate system is constructed from a smoothed bathymetry satisfying the pressure gradient error constraint $\left|H_{i}-H_{i+1}\right| /\left|H_{i}+H_{i+1}\right| \leq$ 0.2 (where $H_{i}$ are the fluid column heights). Then the small scale details of the bathymetry are added back via volume penalization. These local details are effectively represented in a $z$-coordinate system but the smoothing associated with the penalization avoids the staircase effect. Note that the smoothed bathymetry profile must lie below the detail profile (see Fig. 1). In addition, by adjusting the porosity of computational cells near the fluid-solid boundary we can model the subgrid scale geometry of the bathymetry. Because only a relatively small portion of the computational domain is solid, we also avoid unnecessary overhead associated with calculating the solution in the solid regions. Note there is no requirement that the bathymetry be a function of the horizontal coordinates $x$, or even that it be simply connected. The geometry could even be time-dependent.

In the following section we derive the new three-dimensional penalization for a discrete primitive equations model and in Section 3 we validate and illustrate its 


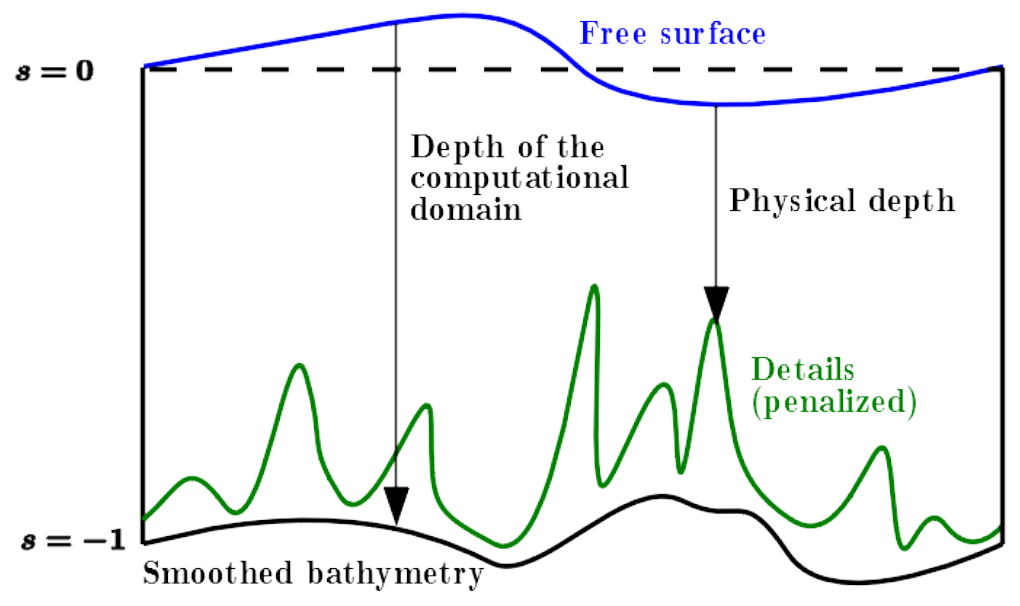

Figure 1: Smoothed bathymetry profile defining the generalized coordinate system (black) and mask defining small scale details (green). The computational domain extends from the surface $s(x, t)=0$ to the smoothed bathymetry $s(x, t)=-1$. The region between the green and black curves is penalized, with porosity $\phi=\alpha \ll 1$.

features by applying it to three standard test cases.

This penalization is straightforward to implement in existing hydrostatic ocean models and be easily extended to moving boundaries, such as melting/freezing ice shelves analogously to Kevlahan and Vasilyev (2005).

\section{Brinkman volume penalization for bathymetry in ocean models}

\subsection{Primitive equations in conservative form}

For simplicity we consider a two-dimensional $x-z$ domain bounded above by the free surface $\eta(x, t)$ and below by the bathymetry $H(x)$. The boundary conditions for the vertical velocity $w(x, z, t)$ at the free surface and bottom are respectively

$$
w(x, \eta, t)=\frac{d z}{d t}=\frac{\partial \eta}{\partial t}+u(x, \eta, t) \frac{\partial \eta}{\partial x} \quad \text { and } \quad w(x,-H, t)=0 .
$$


The horizontal velocity $u(x, z, t)$ satisfies no-slip boundary conditions at the bottom and at any lateral solid boundaries. The primitive equations in conservative form and geopotential $(z)$ vertical coordinate system are:

$$
\begin{aligned}
\frac{\partial u}{\partial t}+\frac{\partial u^{2}}{\partial x}+\frac{\partial u w}{\partial z} & =-g \frac{\partial \eta}{\partial x}-\frac{1}{\rho_{0}} \frac{\partial p_{h}}{\partial x} \\
\frac{\partial u}{\partial x}+\frac{\partial w}{\partial z} & =0 \\
\frac{\partial p_{h}}{\partial z} & =-\rho g \\
\frac{\partial \rho}{\partial t}+\frac{\partial u \rho}{\partial x}+\frac{\partial w \rho}{\partial z} & =0
\end{aligned}
$$

with $p_{h}$ the hydrostatic pressure and $\rho$ the density. Introducing the $s$ generalized vertical coordinate

$$
s(x, z, t)=\frac{z-\eta(x, t)}{H(x)+\eta(x, t)},
$$

and defining $h(x, s, t)=\partial z / \partial s$ we can write down the primitive equations in conservation form as

$$
\begin{aligned}
\frac{\partial h u}{\partial t}+\left.\frac{\partial h u^{2}}{\partial x}\right|_{s}+\frac{\partial h u \Omega}{\partial s} & =-h\left(g \frac{\partial \eta}{\partial x}+\left.\frac{1}{\rho_{0}} \frac{\partial p_{h}}{\partial x}\right|_{s}+\left.\frac{\rho}{\rho_{0}} g \frac{\partial z}{\partial x}\right|_{s}\right), \\
\frac{\partial h}{\partial t}+\left.\frac{\partial h u}{\partial x}\right|_{s}+\frac{\partial h \Omega}{\partial s} & =0, \\
\frac{\partial p_{h}}{\partial s} & =-h\left(\rho-\rho_{0}\right) g \\
\frac{\partial h \rho}{\partial t}+\left.\frac{\partial h u \rho}{\partial x}\right|_{s}+\frac{\partial h \Omega \rho}{\partial s} & =0,
\end{aligned}
$$

where the vertical velocity across moving interfaces $\Omega(x, s, t)$ is defined as

$$
\Omega:=\frac{d s}{d t}=\left.\frac{\partial s}{\partial t}\right|_{z}+\left.u \frac{\partial s}{\partial x}\right|_{z}+w \frac{\partial s}{\partial z}
$$

such that $\Omega$ has homogeneous vertical boundary conditions

$$
\Omega(x, 0, t)=\Omega(x,-1, t)=0 .
$$


In the following, horizontal derivates, unless explicitly stated, are computed along constant $s$.

The total depth is

$$
\int_{-1}^{0} h(x, s, t) \mathrm{d} s=H(x)+\eta(x, t)
$$

and the evolution of the free surface is given by

$$
\frac{\partial(H+\eta)}{\partial t}=\frac{\partial \eta}{\partial t}=-\frac{\partial U}{\partial x}
$$

where $U(x, t)=\int_{-1}^{0} h u \mathrm{~d} s$ is the barotropic flux.

We now discretize the primitive equations (8) vertically into $N$ layers $k=$ $1, \ldots, N$ from the bottom to the free surface, where the thickness of layer $k$ is denoted by $h_{k}(x, t)$,

$$
h_{k}=\int_{s_{k-1 / 2}}^{s_{k+1 / 2}} h(x, s, t) \mathrm{d} s=z_{k+1 / 2}-z_{k-1 / 2} .
$$

This definition of layer thickness ensures that at the discrete level $\sum_{k=1}^{N} h_{k}(x, t)=$ $H(x)+\eta(x, t)$. At the bottom boundary $z_{1 / 2}=-H(x)$ and at the free surface $z_{N+1 / 2}=$ $\eta(x, t)$. Layer thicknesses $h_{k}$ change slightly in time due to the movement of the free surface $\eta(x, t)$ according to

$$
h_{k}(x, t)=h_{k}^{0}(x)\left(1+\frac{\eta(x, t)}{H(x)}\right)
$$

where $\eta(x, 0)=0$. Equation (14) expresses the fact that $N$ vertical layers are distributed between the bottom bathymetry and the moving free surface. Although the layers move slightly due to the motion of the free surface, no vertical remapping is required. 
Discretizing the primitive equations (8) in the vertical direction then gives

$$
\begin{aligned}
\frac{\partial h_{k} u_{k}}{\partial t}+\frac{\partial h_{k} u_{k}^{2}}{\partial x}+\left(\Omega_{k+1 / 2} u_{k+1 / 2}-\Omega_{k-1 / 2} u_{k-1 / 2}\right) & = \\
-h_{k} \frac{\partial}{\partial x}\left(g \eta+\frac{1}{\rho_{0}} p_{k}\right)-h_{k} \frac{\rho_{k}}{\rho_{0}} g \frac{\partial z_{k}}{\partial x} & \\
\frac{\partial h_{k}}{\partial t}+\frac{\partial h_{k} u_{k}}{\partial x}+\left(\Omega_{k+1 / 2}-\Omega_{k-1 / 2}\right) & =0, \\
p_{k+1 / 2}-p_{k-1 / 2} & =-h_{k} \rho_{k} g, \\
\frac{\partial h_{k} \rho_{k}}{\partial t}+\frac{\partial h_{k} u_{k} \rho_{k}}{\partial x}+\left(\Omega_{k+1 / 2} \rho_{k+1 / 2}-\Omega_{k-1 / 2} \rho_{k-1 / 2}\right) & =0 .
\end{aligned}
$$

where $p_{N+1 / 2}=0$ and the discrete vertical velocity $\Omega_{k}$ is integrated over layer $k$, $\Omega_{k}(t)=\int_{s_{k-1 / 2}}^{s_{k+1 / 2}} \Omega(x, s, t) \mathrm{d} s$.

The vertical velocities $\Omega_{k}$ are computed from the continuity equation

$$
\frac{\partial h_{k}}{\partial t}+\frac{\partial h_{k} u_{k}}{\partial x}+\frac{\partial \Omega_{k}}{\partial s}=0
$$

so that

$$
\Omega_{k+1 / 2}=-\sum_{p=1}^{k} \frac{\partial h_{p}}{\partial t}+\frac{\partial h_{p} u_{p}}{\partial x},
$$

where (14) gives the time derivative of $h_{k}$ in terms of $\eta$,

$$
\frac{\partial h_{k}}{\partial t}=\frac{h_{k}^{0}}{H} \frac{\partial \eta}{\partial t}=\frac{h_{k}}{H+\eta} \frac{\partial \eta}{\partial t}
$$

Finally, the evolution equation for the free surface (12) provides an expression for the time derivative of $h_{k}$ to complete the computation of $\Omega_{k+1 / 2}$ in (21)

$$
\frac{\partial h_{k}}{\partial t}=-\frac{h_{k}}{H+\eta} \frac{\partial}{\partial x} \int_{-1}^{0} h u \mathrm{~d} s=\frac{h_{k}}{H+\eta} \frac{\partial}{\partial x} \sum_{p=1}^{N} h_{p} u_{p} .
$$

Next, the horizontal direction is discretized into $M$ columns $i=1, \ldots, M$ of equal size $\Delta x$ and the horizontal fluxes and horizontal gradients are found using 
the standard finite volume approximation and second order central difference approximations respectively. The prognostic variables are layer thicknesses $h_{i k}(t)$, horizontal velocities $u_{i k}(t)$ and layer densities $\rho_{i k}(t)$.

Our goal now is to approximate no-slip boundary conditions for $u_{i k}(t)$ at the bottom and lateral boundaries using Brinkman volume penalization. The continuous and discrete multilayer equations derived in this section conserve total energy (kinetic energy plus gravitational potential energy),

$$
\frac{\partial}{\partial t} \int_{-H}^{\eta} \frac{1}{2} u^{2}+\rho g z \mathrm{~d} z=0
$$

We will see that total energy is also conserved for the penalized equations, apart from the necessary dissipation of kinetic energy in the solid regions associated with their finite permeability.

\subsection{Volume penalized equations}

We proceed by simply extending the volume penalization derived previously for a single shallow water level to multilayer shallow water equations. The main difference is the possibility of using subgrid scale modelling, where the porosity of boundary cells is modified to take into account their subgrid scale structure. This subgrid scale modelling is an alternative to using cut cells or to "staircase" boundaries. We define a new variable $\tilde{h}_{i k}=h_{i k} \phi_{i k}$, where $0 \leq \phi_{i k} \leq 1$ is the porosity of the cell $(i, k)$. Most cells have $\phi_{i k}=1$ (entirely fluid) or $\phi_{i k}=\alpha \ll$ 1 (approximately solid), however cells near the fluid-solid boundary may have 


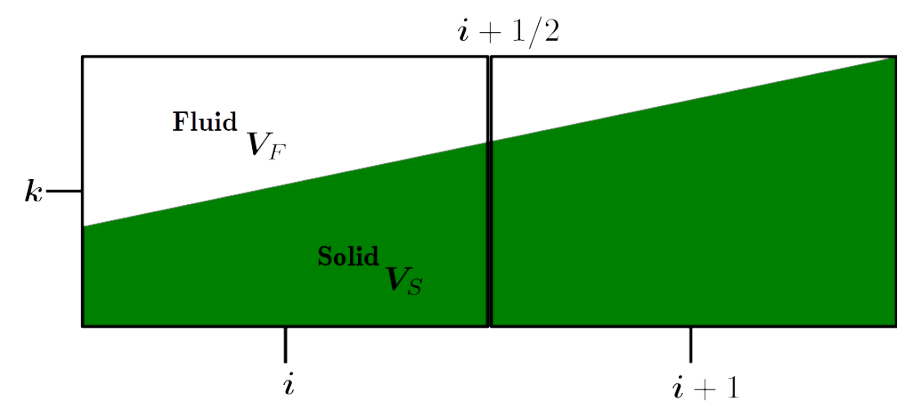

Figure 2: Boundary cells of intermediate porosity $\alpha<\phi_{i k}<1$. The porosity of the cell $\phi_{i k}=$ $\left(V_{F}+\alpha V_{S}\right) /\left(V_{F}+V_{S}\right)$. The green region is the penalized solid.

intermediate porosities depending on how much of the cell is solid,

$$
\phi_{i k}=\frac{V_{F}+\alpha V_{S}}{V_{F}+V_{S}}
$$

where $V_{F}$ is the fluid portion of the cell and $V_{S}$ is the solid portion of the cell, as shown in Fig. 2. The total fluid content in a column is $\sum_{k=1, N} \tilde{h}_{i k}$, which includes the small amount of fluid in any solid regions (controlled by the porosity parameter $\alpha$ ). Note that although the porosity $\phi(x, z)$ does not depend on time, the porosity of a given cell $(i, k)$ does depend on time due to the change in position of the vertical layers due to the motion of the free surface (see equation 14). Therefore the porosity of each cell must be updated at each time step.

The momentum associated with cell $(i, k)$ is $m_{i k}=\tilde{h}_{i k} u_{i k}$. The resistance to flow in a porous medium is determined by its permeability $\sigma(x)=\phi(\mathbf{x}) / \epsilon$, which adds a friction term $-\tilde{h}_{i k} u_{i k} / \epsilon$ to the right hand side of the momentum equation (16). This permeability term is absent in cells that are entirely fluid. For staggered grids (e.g. in finite volume schemes where the velocities are located at cell interfaces $i \pm 1 / 2$ ) $\tilde{h}_{i k}$ must be interpolated appropriately. The interpolation effectively averages the 
porosity of the neighbouring cells $i$ and $i+1 / 2$ (see Fig. 2).

The accuracy of the penalization is controlled by two small parameters $\alpha$ (the porosity of the solid regions) and $\epsilon$ (the permeability of the solid regions). The accuracy of the penalization for a multilevel shallow water model should be the same as for the equivalent single layer shallow water equations considered by Kevlahan et al. (2015), i.e. $O\left(\alpha \epsilon^{1 / 2}\right)$. For the error to converge as $\epsilon \rightarrow 0$ it is necessary to resolve the boundary layer of thickness $O\left(\epsilon^{1 / 2}\right)$ that forms in the solid region adjacent to the fluid-solid boundary. However, one can still obtain a small error with a relatively large value of $\epsilon$ by reducing $\alpha$ (which does not impose any constraints on the grid size or time step).

Penalizing too much of the computational domain wastes computational resources. Therefore, we propose a hybrid approach, starting from a smoothed $\sigma$-coordinate approximation to the true bathymetry. This smoothed bathymetry forms the foundation on which details are added via penalization. The degree of smoothing is the minimum necessary to satisfy the pressure gradient error constraint $\left|H_{i}-H_{i+1}\right| /\left|H_{i}+H_{i+1}\right| \leq 0.2$ (where $H_{i}$ are the fluid column heights). This smoothed bathymetry must be an exterior bound to the true bathymetry (i.e. an envelope) since the fine details of the bathymetry are added by penalization to its interior. This construction of the true bathymetry from the smoothed bathymetry and penalization is shown in Fig. 1. Note that lateral boundaries and overhanging (non-function) features can also be represented via penalization. It is also possible to include moving boundaries (such as melting ice shelves) via penalization. 
Substituting $h=\tilde{h}$ and adding the dissipative Darcy term to the non-penalized equations (16-19) gives the penalized multilayer shallow water equations

$$
\begin{aligned}
\frac{\partial \tilde{h} u}{\partial t}+\frac{\partial \tilde{h} u^{2}}{\partial x}+\frac{\partial \tilde{h} u \Omega}{\partial s} & =-\tilde{h}\left(g \frac{\partial \eta}{\partial x}+\frac{1}{\rho_{0}} \frac{\partial p_{h}}{\partial x}+\frac{\rho}{\rho_{0}} g \frac{\partial z}{\partial x}\right)-\frac{1}{\epsilon} \mathbb{1}(x, z) \tilde{h} u \\
\frac{\partial \tilde{h}}{\partial t}+\frac{\partial \tilde{h} u}{\partial x}+\frac{\partial \tilde{h} \Omega}{\partial s} & =0 \\
\frac{\partial p_{h}}{\partial s} & =-\tilde{h} \rho g \\
\frac{\partial \tilde{h} \rho}{\partial t}+\frac{\partial \tilde{h} u \rho}{\partial x}+\frac{\partial \tilde{h} \Omega \rho}{\partial s} & =0
\end{aligned}
$$

where the indicator function $\mathbb{1}_{i k}=1$ if cell $(i, k)$ includes any solid region and zero otherwise. (We will see that these equations must be supplemented with an evolution equation for $\tilde{h}$ that accounts for the fact that the porosity of a vertical level changes with time as the free surface moves even though the porosity itself is time-independent.)

We now confirm that total energy is conserved, apart from dissipation due to the Darcy term. Considering first the kinetic energy, we have

$$
\frac{\partial}{\partial t} \int_{-H}^{\eta} \frac{1}{2} u^{2} \phi \mathrm{d} z=\int_{-1}^{0} \frac{1}{2} \frac{\partial}{\partial t} \frac{(\tilde{h} u)^{2}}{\tilde{h}} \mathrm{~d} s=\int_{-1}^{0} u \frac{\partial}{\partial t}(\tilde{h} u)-u^{2} \frac{1}{2} \frac{\partial}{\partial t} \tilde{h} \mathrm{~d} s
$$

which has the same form as for the non-penalized equations, but with $h$ replaced by $\tilde{h}$. Using equations (26) and (27) for the time derivatives and imposing the boundary conditions on $\Omega$ gives

$$
\frac{\partial}{\partial t} \int_{-H}^{\eta} \frac{1}{2} u^{2} \phi \mathrm{d} z=-\int_{-1}^{0} \frac{1}{2} \frac{\partial}{\partial x}\left(h u^{3}\right) \mathrm{d} s-\left.\int_{-1}^{0} \tilde{h} u \frac{\partial p_{h}}{\partial x}\right|_{z} \mathrm{~d} s-\frac{1}{\epsilon} \int_{-1}^{0} \mathbb{1}(x, z) \frac{1}{2} \tilde{h} u^{2} \mathrm{~d} s .
$$

The first term on the right hand side is a flux, and therefore its integral must be zero 
on a closed domain. We are therefore left with

$$
\frac{\partial}{\partial t} \int_{-H}^{\eta} \frac{1}{2} u^{2} \phi \mathrm{d} z=-\left.\int_{-1}^{0} \tilde{h} u \frac{\partial p_{h}}{\partial x}\right|_{z} \mathrm{~d} s-\frac{1}{\epsilon} \int_{-1}^{0} \mathbb{1}(x, z) \frac{1}{2} \tilde{h} u^{2} \mathrm{~d} s .
$$

We show below that the gravitational potential energy cancels the second term, leaving only negative definite penalization term.

For the gravitational potential part of the change in total energy (24) we have

$$
\begin{aligned}
\frac{\partial}{\partial t} \int_{-H}^{\eta} \rho g z \phi \mathrm{d} z & =\frac{\partial}{\partial t} \int_{-1}^{0} \rho g z \tilde{h} \mathrm{~d} s=\int_{-1}^{0}-g z\left(\frac{\partial}{\partial x}(u \rho \tilde{h})+\frac{\partial}{\partial s}(\Omega \rho \tilde{h})\right)+\rho g \tilde{h} \frac{\partial z}{\partial t} \mathrm{~d} s \\
& =\int_{-1}^{0} \tilde{h} \rho g u \frac{\partial z}{\partial x}+\tilde{h} \rho g \Omega \frac{\partial z}{\partial s}+\tilde{h} \rho g \frac{\partial z}{\partial t} \mathrm{~d} s .
\end{aligned}
$$

where we have used (29) for the time derivative of $\tilde{h} \rho$ and integrated by parts.

Finally, using the fact that $w=\partial_{t} z+u \partial_{x} z+\tilde{h} \Omega$ we can express the change in gravitational potential energy as

$$
\frac{\partial}{\partial t} \int_{-1}^{0} \rho g z \tilde{h} \mathrm{~d} s=\int_{-1}^{0} \tilde{h} \rho g w \mathrm{~d} s .
$$

This form respects the conversion of potential energy to kinetic energy. We now show that the pressure gradient term in the change in kinetic energy (32) cancels the change in gravitational potential energy. Rewriting the pressure gradient term,

$$
\begin{aligned}
-\left.\int_{-1}^{0} \tilde{h} u \frac{\partial p_{h}}{\partial x}\right|_{z} \mathrm{~d} s & =-\int_{-1}^{0} \tilde{h} u\left(\left.\frac{\partial p_{h}}{\partial x}\right|_{s}+\frac{\partial p}{\partial s} \frac{\partial s}{\partial x}\right) \mathrm{d} s, \\
& =-\int_{-1}^{0}-p_{h} \frac{\partial \tilde{h} u}{\partial x}-\tilde{h} u \rho g \frac{\partial z}{\partial x} \mathrm{~d} s,
\end{aligned}
$$

where we have used

$$
\frac{\partial s}{\partial x}=-\frac{1}{h} \frac{\partial z}{\partial x}
$$

and the hydrostatic relation

$$
\frac{\partial p}{\partial s}=-h \rho g
$$


Now, using the continuity relation (27) with $h=\partial z / \partial s$ we can re-write (35) as

$$
-\left.\int_{-1}^{0} \tilde{h} u \frac{\partial p_{h}}{\partial x}\right|_{z} \mathrm{~d} s=-\int_{-1}^{0} p_{h}\left(\frac{\partial}{\partial t}\left(\phi \frac{\partial z}{\partial s}\right)+\frac{\partial \tilde{h} \Omega}{\partial s}\right)+\tilde{h} u \rho g \frac{\partial z}{\partial x} \mathrm{~d} s .
$$

Since the porosity $\phi$ depends on $z$, but not on time, we have

$$
0=\left.\frac{\partial \phi}{\partial t}\right|_{z}=\left.\frac{\partial \phi}{\partial t}\right|_{s}+\left.\frac{\partial \phi}{\partial s} \frac{\partial s}{\partial t}\right|_{z}
$$

and using

$$
\frac{\partial s}{\partial t}=\frac{1}{h} \frac{\partial z}{\partial t}=\frac{\partial z / \partial t}{\partial z / \partial s}
$$

we get that

$$
\frac{\partial \phi}{\partial t} \frac{\partial z}{\partial s}=\frac{\partial \phi}{\partial s} \frac{\partial z}{\partial t}
$$

which means that $\phi$ and $z$ must satisfy the commutation relation

$$
\frac{\partial}{\partial t}\left(\phi \frac{\partial z}{\partial s}\right)=\frac{\partial}{\partial s}\left(\phi \frac{\partial z}{\partial t}\right)
$$

or, equivalently,

$$
\frac{\partial \tilde{h}}{\partial t}=\frac{\partial}{\partial s}\left(\phi \frac{\partial z}{\partial t}\right)
$$

Equation (38) is a new evolution equation for $\tilde{h}$, replacing (22), which must be integrated in time, and which is not present in the non-penalized equations. It represents the fact that the porosity of a level $k$ changes due to the redistribution of the levels caused by the motion of the free surface.

Now, using the commutation relation (37) in (36) and integrating by parts,

$$
\begin{aligned}
-\left.\int_{-1}^{0} \tilde{h} u \frac{\partial p_{h}}{\partial x}\right|_{z} \mathrm{~d} s & =\int_{-1}^{0} \frac{\partial p_{h}}{\partial s}\left(\phi \frac{\partial z}{\partial t}+\tilde{h} \Omega\right)-\tilde{h} u \rho g \frac{\partial z}{\partial x} \mathrm{~d} s, \\
& =-\int_{-1}^{0} \tilde{h} \rho g\left(\frac{\partial z}{\partial t}+u \frac{\partial z}{\partial x}+h \Omega\right) \mathrm{d} s, \\
& =-\int_{-1}^{0} \tilde{h} \rho g w \mathrm{~d} s .
\end{aligned}
$$


Substituting $-\left.\int_{-1}^{0} \tilde{h} u \partial_{x} p_{h}\right|_{z} \mathrm{~d} s=-\int_{-1}^{0} \tilde{h} \rho g w \mathrm{~d} s$ in the expression for the change in kinetic energy (32) and adding the change in gravitational potential energy (34), the change in total energy becomes

$$
\frac{\partial}{\partial t} \int_{-H}^{\eta} \frac{1}{2} u^{2}+\rho g z \mathrm{~d} z=-\frac{1}{\epsilon} \int_{-1}^{0} \mathbb{1}(x, z) \frac{1}{2} \tilde{h} u^{2} \mathrm{~d} s \leq 0 .
$$

Thus, the penalization is stable since total energy cannot increase. Note that unlike the non-penalized case, total energy is not conserved since some kinetic energy is lost when velocity is damped in the penalized parts of the computational domain. The total loss in kinetic energy is small since the flow penetrates only a small distance $O\left(\epsilon^{1 / 2}\right)$ into the solid regions and the velocity is also small, $O\left(\epsilon^{1 / 2}\right)$.

As for the non-penalized equations, we discretize the primitive equations (2629) vertically into $N$ layers $k=1, \ldots, N$ from the bottom to the free surface, where the thickness of layer $k$ is now

$$
\tilde{h}_{k}=\int_{s_{k-1 / 2}}^{s_{k+1 / 2}} \tilde{h}(x, s, t) \mathrm{d} s .
$$

The penalized discrete equations in each column are therefore

$$
\begin{aligned}
\frac{\partial \tilde{h}_{k} u_{k}}{\partial t}+\frac{\partial \tilde{h}_{k} u_{k}^{2}}{\partial x}+\left(\Omega_{k+1 / 2} u_{k+1 / 2}-\Omega_{k-1 / 2} u_{k-1 / 2}\right) & =-\tilde{h}_{k} \frac{\partial}{\partial x}\left(g \eta+\frac{1}{\rho_{0}} p_{k}+\frac{\rho_{k}}{\rho_{0}} g \frac{\partial z_{k}}{\partial x}\right) \\
-\frac{1}{\epsilon} \mathbb{1}_{i k} \tilde{h}_{k} u_{k}, & \\
p_{k+1 / 2}-p_{k-1 / 2} & =-\tilde{h}_{k} \rho_{k} g, \\
\frac{\partial \tilde{h}_{k} \rho_{k}}{\partial t}+\frac{\partial \tilde{h}_{k} u_{k} \rho_{k}}{\partial x}+\left(\Omega_{k+1 / 2} \rho_{k+1 / 2}-\Omega_{k-1 / 2} \rho_{k-1 / 2}\right) & =0, \\
\frac{\partial \tilde{h}_{k}}{\partial t}=\phi\left(z_{k+1 / 2}\right)\left(\frac{\partial z}{\partial t}\right)_{k+1 / 2}-\phi\left(z_{k-1 / 2}\right)\left(\frac{\partial z}{\partial t}\right)_{k-1 / 2}, &
\end{aligned}
$$

where the last equation enforces the constraint (38) and is advanced in time using 
an Euler step. Note that the vertical velocities $\Omega_{k+1 / 2}$ are still deduced from the continuity equation as $\Omega_{k+1 / 2}=\Omega_{k-1 / 2}-\left(\partial_{t} \tilde{h}_{k}+\partial_{x}\left(\tilde{h}_{k} u_{k}\right)\right)$, but with $\partial_{t} \tilde{h}_{k}$ now given by (45) instead of (22).

For consistency (or constancy preservation) we need to show that setting $\rho_{k}=1$ in equation (44) and summing over all vertical layers gives the equation for the motion of the free surface $\partial_{t} \eta+\partial_{x} U=0$, where $U=\sum_{k=1}^{N} \partial_{x} \tilde{h}_{k} u_{k}$, equivalent to (12). This requires showing that $\sum_{k=1}^{N} \partial_{t} \tilde{h}_{k}=\partial_{t} \eta$. Taking the time derivative of (41) and using the commutation relation (38) gives

$$
\begin{aligned}
\frac{\partial \tilde{h}_{k}}{\partial t} & =\int_{s_{k-1 / 2}}^{s_{k+1 / 2}} \frac{\partial \tilde{h}_{k}}{\partial t} \mathrm{~d} s, \\
\frac{\partial \tilde{h}_{k}}{\partial t} & =\int_{s_{k-1 / 2}}^{s_{k+1 / 2}} \frac{\partial}{\partial s}\left(\phi \frac{\partial z}{\partial t}\right) \mathrm{d} s, \\
& =\phi\left(s_{k+1 / 2}\right)\left(\frac{\partial z}{\partial t}\right)_{k+1 / 2}-\phi\left(s_{k-1 / 2}\right)\left(\frac{\partial z}{\partial t}\right)_{k-1 / 2} .
\end{aligned}
$$

Now, summing over $k$,

$$
\frac{\partial}{\partial t} \sum_{k=1}^{N} \tilde{h}_{k}=\phi\left(s_{N+1 / 2}\right)\left(\frac{\partial z}{\partial t}\right)_{N+1 / 2}-\phi\left(s_{1 / 2}\right)\left(\frac{\partial z}{\partial t}\right)_{1 / 2}=\frac{\partial \eta}{\partial t}
$$

as required, where we have assumed that $\phi\left(s_{N+1 / 2}\right)=1$ (the top layer is entirely fluid).

In the following section we verify the accuracy and performance of the penalization on three standard two-dimensional test cases. The new penalized equations (42-45) correspond precisely to the usual model equations for $h_{k}$. The only modifications required are changing the definition of $h_{k}$ to account for porosity, solving an additional equation for the evolution of $\tilde{h}_{k}(45)$ and adding the friction term 
representing permeability to the momentum equation (42). This makes it very straightforward to add penalization to an existing code.

\section{Validation and examples}

In this section we specify the computational ocean model in which the Brinkman penalization is tested, and explain how the permeability and porosity functions defining the penalization were implemented numerically. Finally, we report the results for two idealized test cases: upwelling and internal tides.

\subsection{Computational model}

The Brinkman volume penalization is implemented in the CROCO ocean model (Debreu et al., 2012; Shchepetkin and McWilliams, 2005). This model solves the three-dimensional free surface primitive equations using an Arakawa C-grid in the horizontal direction and terrain following coordinates in the vertical direction. Although the original version of CROCO uses time splitting for the treatment of the fast (barotropic) mode, for simplicity we use it here in its non-split version where barotropic and baroclinic time steps are equal.

\subsection{Choice of the Brinkman penalization parameters}

The fluid-solid interface must be smoothed over a few grid points to avoid the staircase effect and to maintain numerical stability. The choices presented here have proven to be robust in the two test cases we consider here. 
The mask $\mathbb{1}(r)$ defining the solid regions is first replaced by a mask $\mathbb{1}_{S}(r)$ which has been smoothed vertically using a tanh function,

$$
\mathbb{1}_{S}(r)=\frac{1}{2}\left(1+\tanh \lambda\left(r-z_{0}\right)\right), \text { with } r=\frac{\left(z-z_{\text {bottom }}\right)}{\Delta z} .
$$

The parameters $\lambda$ and $z_{0}$ are chosen so that $\mathbb{1}_{S}(-1 / 2)=1 / 25$ and $\mathbb{1}_{S}(1 / 2)=2 / 5$ (i.e. $\left.\lambda \approx 1.38629, z_{0} \approx 0.646241\right)$. The shape of $\mathbb{1}_{S}(r)$ is shown in Fig. 3 . Note that $\mathbb{1}_{S}(r)$ is not symmetric with respect to the fluid-solid interface, so that the penalized part of the fluid region is minimized. The permeability $\sigma(r)=\mathbb{1}_{S}(r) / \epsilon$ therefore varies smoothly between 0 and $1 / \epsilon$ across the fluid-solid interface in the vertical direction. The permeability $\sigma$ has the value $1 /(25 \epsilon)$ at the first fluid grid

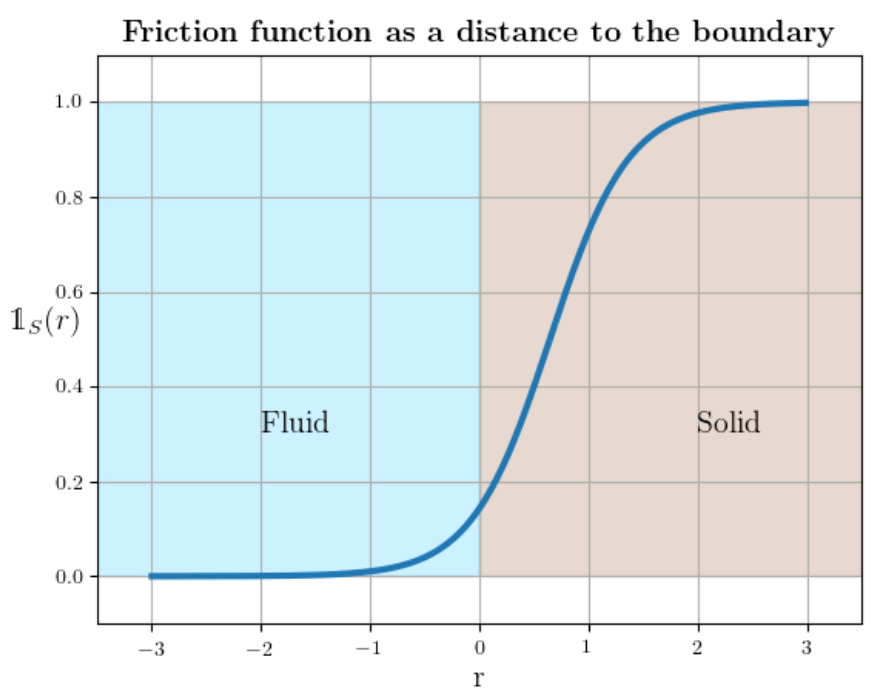

Figure 3: Function $\mathbb{1}_{S}(r)$ used to smooth the porosity and permeability at the fluid-solid interface in the vicinity of topographical features.

point above the bathymetry and the value $2 /(5 \epsilon)$ at the first point inside the solid region. These discrete $\sigma$ values are then smoothed using two passes of a multidi- 
mensional Shapiro filter Shapiro (1970) with parameters $(1 / 4,1 / 2,1 / 4)$, first in the horizontal direction and then in the vertical direction. The final mask $\mathbb{1}_{S}(r)$ is therefore smoothed in the vertical, horizontal and diagonal directions. The smoothed porosity is then found by subsituting $\mathbb{1}_{S}(r)$ in equation (4).

In the next two examples the permeability parameter $\epsilon=4 \Delta t$ and the porosity parameter $\alpha=10^{-2}$. These values have been found to lead to robust and accurate simulations as shown below. (Recall that we require $\epsilon<\Delta t$ for stability, there is no stability constraint on $\alpha$, and that the error in the penalized boundary condition is $O\left(\alpha \epsilon^{1 / 2}\right)$.) In these preliminary experiments, we do not take advantage of subgrid scale modelling of the bathymetry. In practice, this means that we assume that the bathymetry within a cell is constant (i.e. zero slope in Fig. 2).

The modified layer thicknesses $\tilde{h}$ are initialized using the porosity coefficient $\alpha$ as $\tilde{h}(t=0)=\max \left(\alpha h(t=0), \Delta z_{\min }\right)$ where $\Delta z_{\min }$ is a minimum layer thickness (set here to $\Delta z_{\min }=0.1 \mathrm{~m}$ and used to prevent negative $\tilde{h}$ ) and are then time integrated using (45).

\subsection{Upwelling}

The first test case assesses the physical consistency of bottom flow represented with a $z$-coordinate based penalization. Our goal is to evaluate whether the penalization eliminates the usual spurious "staircase" gravity flow due to the $z$-coordinate representation of sloping bathymetry. We also measure the error of the penalized solution as a function of the control parameters $\epsilon$ and $\alpha$.

We consider two-dimensional cross-shore coastal upwelling flow driven by an 
along-shore wind stress $\tau_{y}=0.07 \mathrm{~Pa}$ on an $f$-plane (at a latitude of $21^{\circ} \mathrm{S}$ ) with constant slope bathymetry. A steady geostrophic onshore flow of $u_{G}=2 \mathrm{~cm} / \mathrm{s}$ is imposed by a constant alongshore pressure gradient using the geostrophic balance $\left.-g \partial_{y} \eta=f u_{G}\right)$. The vertical viscosity coefficient is $K_{V}=10^{-3} \mathrm{~m}^{2} / \mathrm{s}$. The model is linear (no momentum advection) and there is no horizontal viscosity.

This configuration has a steady state with a known analytical solution derived as a two-dimensional extension of classical one-dimensional Ekman theory (Estrade et al., 2008; Marchesiello and Estrade, 2010). The numerical solutions are obtained as instantaneous fields after 20 days of spin up, which is sufficient to reach the steady state.

The width of the horizontal domain is $200 \mathrm{~km}$ and is discretized with 200 cells, leading to a horizontal resolution $\Delta x=1 \mathrm{~km}$. The exact bathymetry is given by the linear water depth function

$$
h(x)=\frac{h_{\max }\left(x_{l}-x\right)+h_{\min } x}{x_{l}},
$$

with $h_{\min }=4 \mathrm{~m}, h_{\max }=205 \mathrm{~m}, x_{l}=200 \mathrm{~km}$ and is shown in Fig. 4 . The vertical grid has 80 equally spaced levels and the time step is $\Delta t=10.5 \mathrm{~s}$.

For this experiment, a generalized terrain-following $\sigma$ vertical coordinate is obviously a very good choice since the bathymetry is smooth and the vertical resolution increases naturally near the shore where the water depth is very small. Fig. 5 compares the analytic and numerical $\sigma$-coordinate solutions after 20 days in the rightmost part of the domain where $h / D \leq 2.5\left(D=\pi \sqrt{2 K_{V} /|f|} \approx 20 \mathrm{~m}\right.$ is the Ekman layer depth). The numerical solution using $\sigma$-coordinates is clearly very 


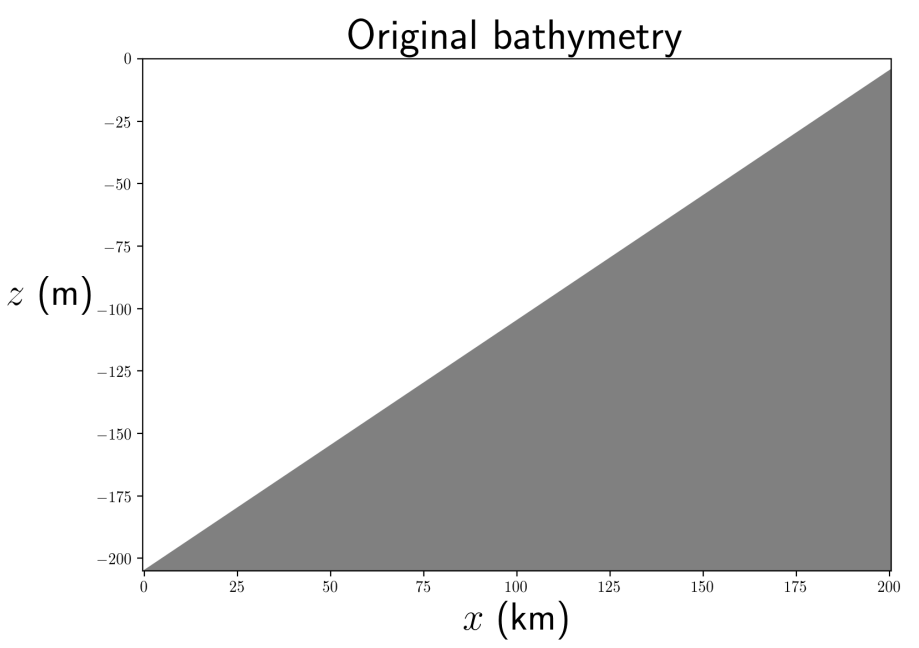

Figure 4: Exact linear bathymetry for the upwelling test case.

accurate, both qualitatively and quantitatively, for this test case.

We now examine solutions obtained with volume penalization and a vertical $z$ coordinate. The results are, of course, sensitive to the choice of the base bathymetry (i.e. the smoothed bathymetry in Fig. 1) which determines how much of the domain is penalized. The larger the penalized area, the coarser the resolution of the fluid region since the vertical levels are now distributed between the base bathymetry and the free surface. A small number of vertical levels in the fluid region clearly limits the accuracy of both the penalization and the representation of the nearshore solution.

This test case has two main objectives. First, to see if the penalization is able to eliminate the spurious staircases effect associated with the $z$-like vertical coordinates. Secondly, to verify that the penalized solution converges to the terrain following $\sigma$-coordinate solution as the penalized portion of the domain decreases. 
We performed three experiments with different values of depths $h_{\min }$ of the computational domain at the inshore side of the domain, and thus different depths of penalization of the solid region (n.b. the actual inshore water depth is fixed at $4 \mathrm{~m})$. Depths $h_{\min }=h_{\max }=205 \mathrm{~m}$ corresponds to a flat base bathymetry where the entire solid region shown in Fig. 4 is penalized. This case is very poorly resolved at the shore, where it has only two vertical fluid levels. The two others experiments penalize smaller depths of the solid: $h_{\min }=50 \mathrm{~m}$ and $h_{\min }=10 \mathrm{~m}$, and have much better vertical resolution near the shore. The corresponding bathymetry profiles are shown on Fig. 6, together with the associated vertical grids.

As shown qualitatively in Fig. 7, and quantified in Table 1, the accuracy of the penalized simulations converges to the accuracy of the non-penalized simulation as the depth of the penalized part of the computational domain decreases. This is primarily due to the increase in the vertical resolution of the fluid near the shore as the depth of the penalized region decreases. In addition, there is no sign of spurious "staircase" flow in any of the the penalized solutions. This test has therefore confirmed the qualitative and quantitative accuracy of the volume penalization.

We now examine at the sensitivity of the results to the choice of penalization and porosity control parameters for the experiment with $h_{\min }=50 \mathrm{~m}$. Fig. 8 shows the root mean square errors (RMSE) for different values of $\epsilon$ and $\alpha$. When the friction coefficient is too large (i.e. the permeability parameter $\epsilon$ is too small), the results are largely insensitive to the size of the porosity coefficient $\alpha$. This is because if $\epsilon$ is too small the the skin depth layer $\delta=O(\sqrt{v \epsilon})$ associated with the 
Flat $\left(h_{\min }=205 \mathrm{~m}\right) \quad h_{\min }=50 \mathrm{~m} \quad h_{\min }=10 \mathrm{~m} \quad \sigma\left(h_{\min }=4 \mathrm{~m}\right)$

\begin{tabular}{lcccc}
\hline Geostrophic velocity $v_{g}(\mathrm{~cm} / \mathrm{s})$ & 6.03 & 1.62 & 1.26 & 0.93 \\
Alongshore velocity $v(\mathrm{~cm} / \mathrm{s})$ & 6.22 & 1.53 & 0.78 & 0.56 \\
Streamfunction $\psi(\%)$ & 3.43 & 1.41 & 1.06 & 1.19 \\
\hline
\end{tabular}

Table 1: Root mean square errors for the upwelling test case for the three penalized depths $h_{\min }$, together with the unpenalized $\sigma$-coordinates computation. In all penalized cases $\epsilon=4 \Delta t$ and $\alpha=$ 0.01. The accuracy of the penalized simulations converges to the accuracy of the non-penalized simulation as the depth of the penalized part of the computational domain decreases.

permeability penalization is not properly resolved, leading to qualitatively inaccurate results (e.g. oscillations). Conversely, when $\epsilon$ is not too small the skin depth is well-resolved, and the error can be controlled effectively by the porosity parameter $\alpha$ alone. In this test case $v=K_{v}=10^{-3} \mathrm{~m}^{2} / \mathrm{s}$ and if $\epsilon=12 \Delta t=126 \mathrm{~s}$, for a Heaviside mask the skin depth $\delta \approx 1.8 \mathrm{~m}$. In the region of interest $h / D>-2.5 \mathrm{~m}$ the vertical grid size is $0.625 \mathrm{~m} \leq \Delta z \leq 1.25 \mathrm{~m}$ and so the skin depth is resolved with between one and three grid points. In practice, since we use the smoothed mask (48), the skin depth is two or three grid points larger and is in fact better resolved.

\subsection{Internal tides}

This test case is inspired by Di Lorenzo et al. (2006), which focuses on energy estimates of $M_{2}$ tidal conversion at steep oceanic ridges. As mentioned in this paper, the smoothing required by the terrain-following $\sigma$ vertical coordinates negatively affects the tidal conversion. The objective of this test case is thus to see 
if the Brinkman penalization is able to correctly represent the bathymetry details of the oceanic ridges, and so to improve the computation of energy conversion. As in Di Lorenzo et al. (2006), an $M_{2}$ tide is generated by adding a body force in the horizontal momentum equations, $B_{u}(t)=\omega U_{0} \cos (\omega t), B_{v}(t)=f U_{0} \sin (\omega t)$ which results (from a flat bottom) in an external velocity with $U=U_{0} \sin (\omega t), V=0 \mathrm{~cm} / \mathrm{s}$. The frequency of the $M_{2}$ tide is $\omega=2 \pi /(12.4 h)$ and $U_{0}=2 \mathrm{~cm} / \mathrm{s}$. The domain is periodic in the $x$ direction and the integration time corresponds to 12 tidal cycles.

The idealized bathymetry $h(x)$ is given by the following expression and is shown on Fig. 9

$$
h(x)=\frac{1}{3} H \sin \left(\pi \frac{x}{L}\right)+H_{1}\left(e^{-\left(\left(x-x_{-1}\right) / s\right)^{2}}+e^{-\left(\left(x-x_{1}\right) / s\right)^{2}}\right)+H_{0}+e^{-\left(\left(x-x_{0}\right) / s\right)^{2}}
$$

with $H_{1}=\frac{12}{45} H, H_{0}=\frac{6}{10} H, x_{-1}=L / 2-L / 6, x_{1}=L / 2+L / 6, x_{0}=L / 2$ and $s=L / 150$.

The $\sigma$-coordinates must be smoothed to satisfy the pressure gradient error criterion

$$
r_{\max }=\max _{i} \frac{\left|h_{i+1}-h_{i}\right|}{h_{i}+h_{i+1}} \leq 0.2
$$

The models are run at two different horizontal resolutions $\Delta x=3 \mathrm{~km}$ and $6 \mathrm{~km}$, with and without penalization. Both runs have $40 \sigma$ vertical levels. The $3 \mathrm{~km} \sigma$ coordinate run does not require smoothing to reduce the pressure gradient error and so it is our reference simulation. The $6 \mathrm{~km} \sigma$-coordinate run requires four smoothing passes (using a Shapiro filter) to satisfy the smoothing criterion for the $\sigma$ vertical coordinates. The corresponding bathymetry is shown in black on Fig. 10. 
In addition to these $\sigma$-coordinate runs, two penalized simulations are performed. The first penalization run is at $6 \mathrm{~km}$ resolution with the smoothed bathymetry as the base bathymetry and fine scales added via penalization. The second penalization run is at $3 \mathrm{~km}$ resolution with the same base bathymetry as the $6 \mathrm{~km}$ run. This last simulation allows us to separate the errors linked to bathymetry representation and horizontal resolution.

According to the linear theory of internal waves (e.g. Laurent et al. (2003)), the ratio $\mu$ between the horizontal $k_{x}$ and vertical $k_{z}$ wavenumbers is

$$
\mu=\left|\frac{k_{x}}{k_{z}}\right|=\sqrt{\frac{\omega^{2}-f^{2}}{N^{2}-\omega^{2}}},
$$

where $\omega$ is the frequency of the $M 2$ tide $(\omega=2 \pi / T, T=12.4 \mathrm{~h}), f$ is the Coriolis parameter $\left(f=10^{-4} \mathrm{~s}^{-1}\right)$ and $N$ is the Brunt Väisälä frequency $\left(N=2 \times 10^{-3} \mathrm{~s}^{-1}\right)$. This leads to $\mu \approx 0.05$. The horizontal wavelength $\lambda_{x}^{n}$ of the baroclinic mode $n$ is

$$
\lambda_{x}^{n}=\frac{1}{\mu} \lambda_{z}^{n}, \text { with } \lambda_{z}^{n}=\frac{2 H}{n} \quad\left(k_{z}=\frac{n \pi}{H}\right)
$$

With $H \approx 2750 \mathrm{~m}$ (the depth at the foots of the ridges), we get

$$
\lambda_{x}^{n}=\frac{1}{0.05} \frac{5550}{n}=\frac{111000}{n} .
$$

Assuming that the effective resolution of the numerical model is approximately $8 \Delta x$, we deduce than for the high resolution $\left(\Delta_{x}=3 \mathrm{~km}\right)$ run four to five baroclinic modes can be correctly represented, while for the coarse resolution $\left(\Delta_{x}=6 \mathrm{~km}\right)$ run, only the first two or three baroclinic modes can be correctly represented. Thus, even if the penalized solution perfectly captured the dynamics of the small scale bathymetry features, it could capture at most three baroclinic modes. 
The instantaneous solutions of the three simulations at the end of the 12 tidal cycles are shown in Fig. 11. As explained above, the coarse resolution $6 \mathrm{~km}$ runs cannot represent more than the the first two or three baroclinic modes. This explains the larger pattern of internal tides beams for the $6 \mathrm{~km}$ simulations (see Fig. 11 $\mathrm{b}$ and c) compared to the $3 \mathrm{~km}$ resolution reference simulation. Nevertheless, the $6 \mathrm{~km}$ penalized run has higher amplitude velocity fluctuations than the nonpenalized run, and thus shows more intense internal tides closer in amplitude to the reference $6 \mathrm{~km}$ run than the non-penalized $6 \mathrm{~km}$ run. Because the Brinkman volume penalization more accurately represents the small scale structure of the ridges, it produces a more accurate (larger) tidal conversion rate between barotropic and baroclinic mechanical energy. The penalized fine resolution $3 \mathrm{~km}$ run is extremely close to the reference unpenalized $3 \mathrm{~km}$ run. In fact, the beams appear to be even better defined.

For the fine resolution $3 \mathrm{~km}$ simulations, with and without penalization, we also carried out a pressure gradient error test. Indeed, the main objective of the the Brinkman penalization approach is to reduce the pressure gradient error associated with $\sigma$-coordinates. In these simulations the barotropic $M_{2}$ forcing is removed and, since the initial temperature field is constant, the velocity fields should remain zero. The criterion for acceptable pressure gradient error is $r_{\max } \leq 0.2$ (49), and we have set $r_{\max }=0.1$ for the (smooth) base bathymetry of the penalized run. Fig. 12 shows the $u$ velocity component after $12 M_{2}$ tidal cycles. The pressure gradient errors remain relatively small in both these idealized simulations (maximum abso- 
lute values of $u$ are less than $\left.10^{-6} \mathrm{~m} / \mathrm{s}\right)$. However, as expected, the errors are even smaller in the penalization run.

\section{Conclusions}

We have extended the Brinkman volume penalization introduced by Kevlahan et al. (2015) for coastlines in the shallow water equations to topography in the three-dimensional hydrostatic primitive equations. In practice, the penalization is used to add small scale topographical details to a smoothed terrain vertical coordinate system. This approach combines the advantages of both $z$-coordinates and $\sigma$ terrain-following coordinates while avoiding their respective drawbacks. This penalization was easily implemented in the CROCO ocean model (Debreu et al., 2012; Shchepetkin and McWilliams, 2005) with only minimal changes (i.e. an additional source term in the equations for the horizontal velocities and a new evolution term for the layer thicknesses). We explained in detail how to choose the parameters $\alpha$ and $\epsilon$ controlling the accuracy of the penalized topography, as well as how the topography must be smoothed over a few grid points near the fluidsolid transition region. We also discussed how the porosity $\alpha$ of a grid cell can be chosen to model some unresolved features of the subgrid scale topography. Since the error of the penalization is $O\left(\epsilon^{1 / 2} \alpha\right)$, an appropriate choice of the porosity $\alpha$ in the solid regions allows for larger values of the velocity friction parameter $\epsilon$, and hence a less restrictive time step and larger grid size (the value of $\epsilon$ places stability restrictions on the time step and accuracy restrictions on the horizontal and vertical 
grids).

The new Brinkmann volume penalization was applied to two challenging twodimensional test cases: coastal upwelling (Estrade et al., 2008; Marchesiello and Estrade, 2010) and internal tides near highly peaked oceanic ridges (Di Lorenzo et al., 2006). The upwelling test case confirmed that the penalization completely eliminates the staircase effect associated with $z$-coordinates and that the numerical penalized solutions converge to the analytical solutions (and the generalized $\sigma$ coordinate numerical solutions) as the penalized portion of the solid decreases, allowing more vertical levels in the inshore fluid region. In the internal tides experiment, the bathymetry smoothing necessary for the $\sigma$-coordinates strongly suppresses the high frequency baroclinic modes. However, when the small scale bathymetry details are added back using volume penalization these high frequency modes are recovered.

For simplicity, in this first validation work we implemented the penalization in a single time step (non split) version of CROCO. Our immediate next step is to implement the penalization in the standard version of CROCO, where the fast barotropic and slow baroclinic modes are advanced using appropriate fast and slow time steps. This will allow us to perform more realistic validations on threedimensional test cases for long runs.

There are many interesting potential applications of this new topography penalization. For example, more sophisticated subgrid modelling (following Adcroft (2013)), ice shelf modelling, wetting and drying. We also imagine taking advan- 
tage of the physical porous medium equations underlying the penalization to better model cases such as marshes, mud or coastal inundation that are naturally porous medium flows.

\section{Acknowledgments}

NKRK gratefully acknowledges funding from Université Grenoble-Alpes, CNRS and NSERC. LD acknowledges support by the ANR through contract ANR-14CE23-0010 (HEAT).

\section{References}

Adcroft, A., 2013. Representation of topography by porous barriers and objective interpolation of topographic data. Ocean Modelling 67, 13-27.

Angot, P., Bruneau, C.H., Fabrie, P., 1999. A penalization method to take into account obstacles in viscous flows. Numerische Mathematik 81, 497-520.

Bao, J., Chow, F.K., Lundquist, K.A., 2018. Large-Eddy Simulation over Complex Terrain Using an Improved Immersed Boundary Method in the Weather Research and Forecasting Model. Monthly Weather Review 146, 2781-2797.

Debreu, L., Marchesiello, P., Penven, P., Cambon, G., 2012. Two-way nesting in split-explicit ocean models: Algorithms, implementation and validation. Ocean Modelling 49-50, 1-21. 
Di Lorenzo, E., Young, W.R., Smith, S.L., 2006. Numerical and analytical estimates of $\mathrm{m} 2$ tidal conversion at steep oceanic ridges. Journal of Physical Oceanography $36,1072-1084$.

Estrade, P., Marchesiello, P., de Verdiere, A.C., Roy, C., 2008. Cross-shelf structure of coastal upwelling: a two-dimensional expansion of ekman's theory and a mechanism for innershelf upwelling shutdown. J. Marine Research 66, 589616.

Ghasemi, A., Kevlahan, N.K.R., 2017. The role of Reynolds number in the fluidelastic instability of tube arrays. J. Fluids Structures 73, 16-36.

Kevlahan, N., Ghidaglia, J.M., 2001. Computation of turbulent flow past an array of cylinders using a spectral method with brinkman penalization. Eur. J. Mech./B $20,333-350$.

Kevlahan, N., Vasilyev, O., 2005. An adaptive wavelet collocation method for fluid-structure interaction at high Reynolds numbers. SIAM J. Sci. Comput. 26, $1894-1915$.

Kevlahan, N.K.R., 2007. Three-dimensional Floquet stability analysis of the wake in cylinder arrays. J Fluid Mech. 592, 79-88. doi:10.1017/ S0022112007008798.

Kevlahan, N.K.R., 2011. The role of vortex wake dynamics in the flow-induced vibration of tube arrays. J. Fluids Struct. 27, 829-837. 
Kevlahan, N.K.R., Dubos, T., Aechtner, M., 2015. Adaptive wavelet simulation of global ocean dynamics using a new Brinkman volume penalization. Geoscientific Model Development 8, 3891-3909. doi:\{10.5194/gmd-8-3891-2015\}.

Laurent, L.S., Stringer, S., Garrett, C., Perrault-Joncas, D., 2003. The generation of internal tides at abrupt topography. Deep Sea Research Part I: Oceanographic Research Papers 50, 987 - 1003.

Lundquist, K.A., Chow, F.K., Lundquist, J.K., 2010. An Immersed Boundary Method for the Weather Research and Forecasting Model. Monthly Weather Review 138, 796-817.

Lundquist, K.A., Chow, F.K., Lundquist, J.K., 2012. An Immersed Boundary Method Enabling Large-Eddy Simulations of Flow over Complex Terrain in the WRF Model. Monthly Weather Review 140, 3936-3955.

Marchesiello, P., Debreu, L., Couvelard, X., 2009. Spurious diapycnal mixing in terrain-following coordinate models: The problem and a solution. Ocean Modelling 26, 156-169.

Marchesiello, P., Estrade, P., 2010. Upwelling limitation by geostrophic onshore flow. J. Marine Research 68, 37-62.

Mellor, G.L., Ezer, T., Oey, L.Y., 1994. The pressure gradient conundrum of sigma coordinate ocean models. Journal of Atmospheric and Oceanic Technology 11, $1126-1134$. 
Peskin, C.S., 1972. Flow patterns around heart valves: a numerical method. J. Comput. Phys. 10, 252-271.

Reckinger, S., Vasilyev, O., Fox-Kemper, B., 2012. Adaptive volume penalization for ocean modeling. Ocean Dyn. 62, 1201-1215.

Shapiro, R., 1970. Smoothing, Filtering, and Boundary Effects. Reviews of Geophysics and Space Physics 8, 359. doi:10.1029/RG008i002p00359.

Shchepetkin, A.F., McWilliams, J.C., 2005. The regional oceanic modeling system (ROMS): a split-explicit, free-surface, topography-following-coordinate oceanic model. Ocean Modelling 9, 347-404. 

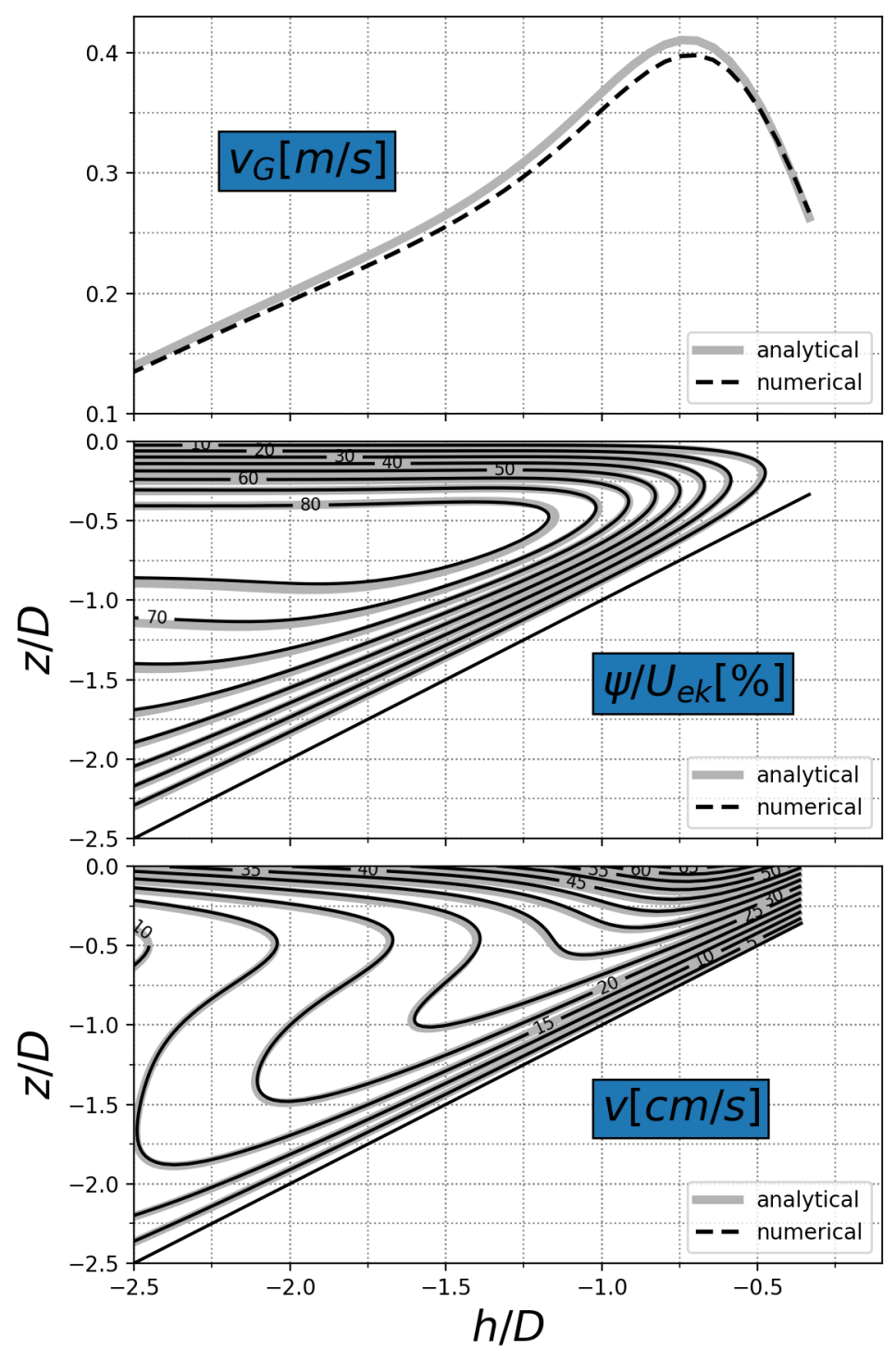

Figure 5: Comparison of the exact analytical and unpenalized numerical solutions using terrainfollowing $\sigma$-coordinates for the upwelling test case after 20 days. The horizontal axis gives the distance from the shore in terms of the local normalized depth $(h / D=-2.5$ corresponds to $x \approx$ $154 \mathrm{~km})$. Top: the alongshore geostrophic current $v_{G}$. Middle: the cross-shore streamfunction $\phi$ normalized by the Ekman transport $U_{E K}=\left|\tau_{y} / \rho_{0} f\right|$. Bottom: the total alongshore velocity $v$. 

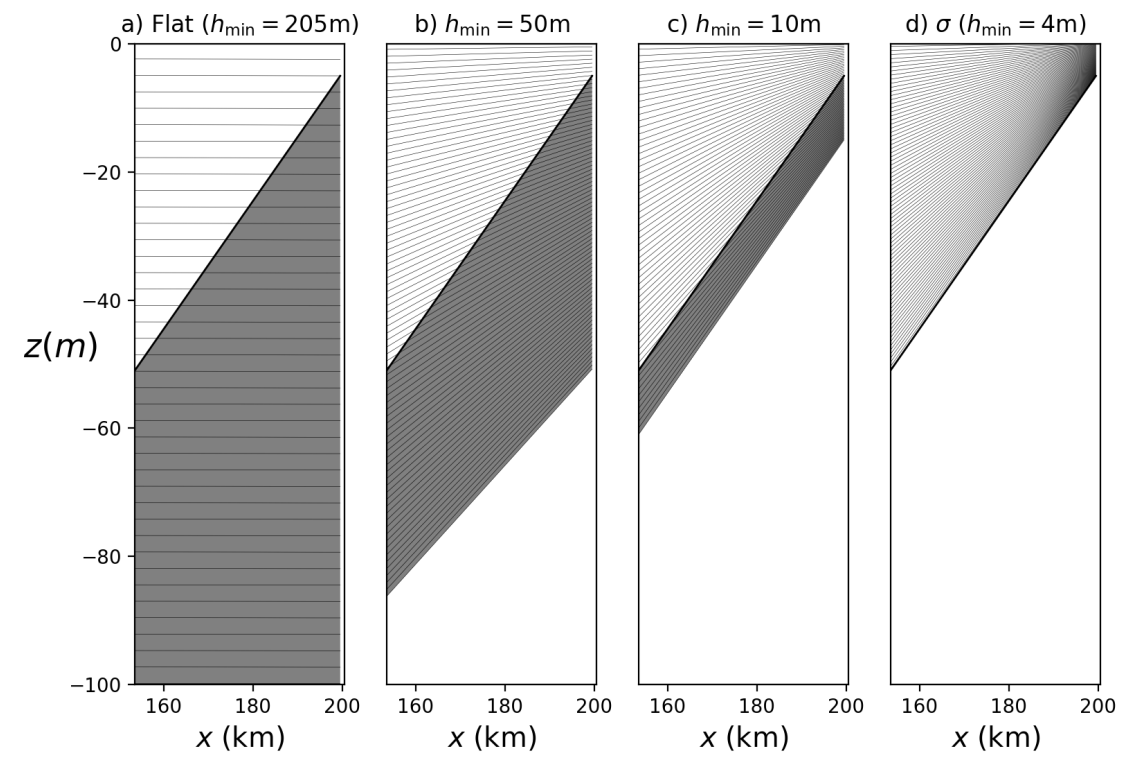

Figure 6: Penalized part of the domain (in grey) and associated vertical levels for three different depths $h_{\min }$ of the penalized region, together with the terrain-following $\sigma$-coordinate case (rightmost figure). Only the first $100 \mathrm{~m}$ below the surface is shown. Note that there are vertical levels in the penalized part of the solid region. In the leftmost figure the entire solid region of Fig. 4 is penalized, while the rightmost figure has no penalization ( $\sigma$-coordinates). 

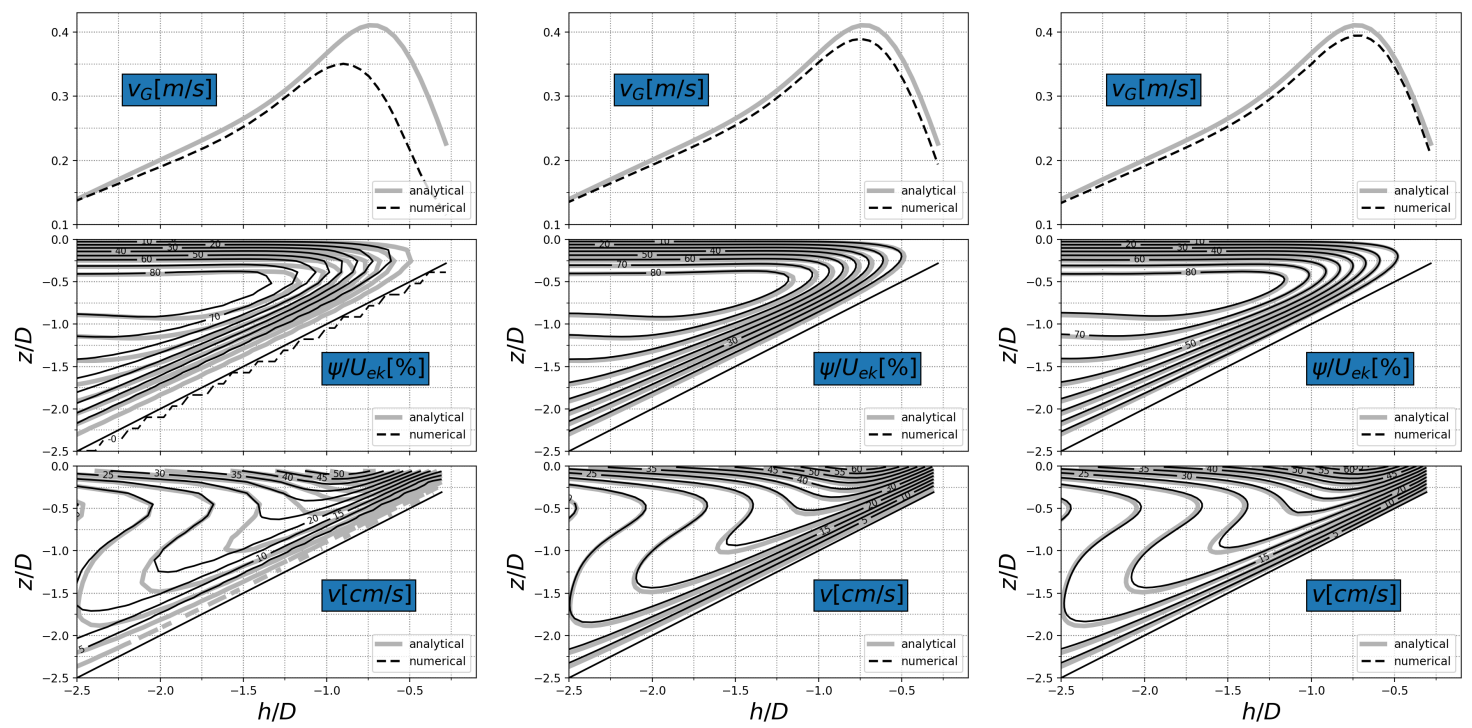

Figure 7: Results obtained for penalized regions of depths $h_{\min }=205 \mathrm{~m}, 50 \mathrm{~m}$ and $10 \mathrm{~m}$ (left to right) for the upwelling test cases. The horizontal axis gives the distance from the shore in terms of the local normalized depth $(h / D=-2.5$ corresponds to $x \approx 154 \mathrm{~km})$. In all cases $\epsilon=4 \Delta t$ and $\alpha=0.01$. As expected, the numerical penalized results converge to the analytical results as the depth of the penalized region decreases (see also table 1). 

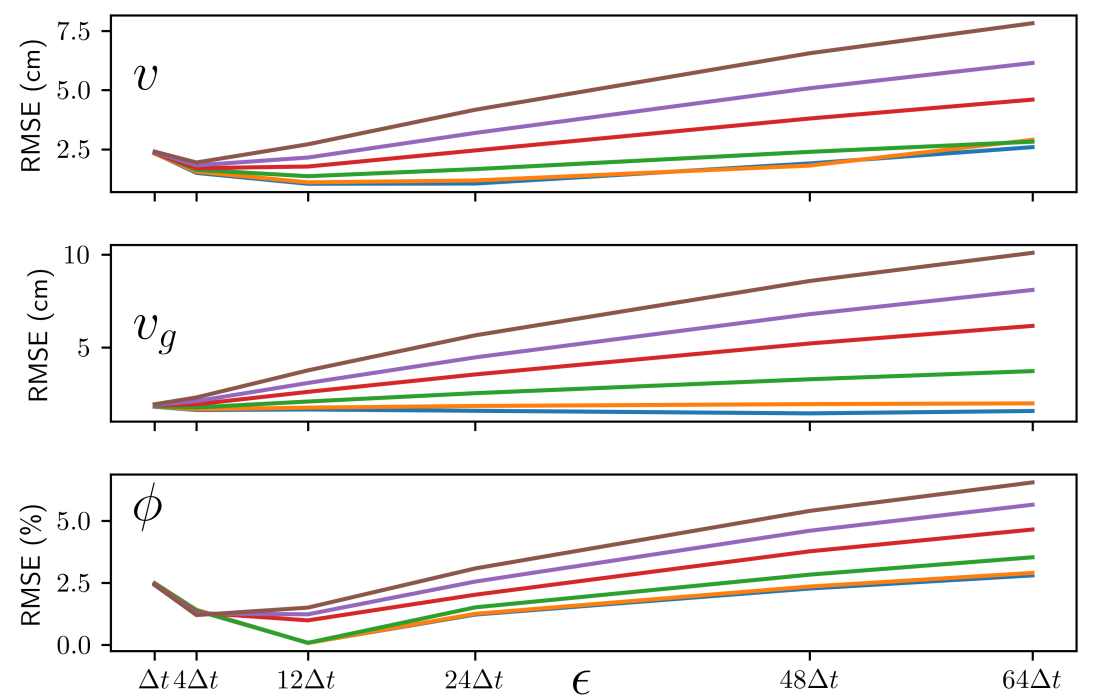

$\longrightarrow \alpha=0.01 \longrightarrow \alpha=0.1 \longrightarrow \alpha=0.25 \longrightarrow \alpha=0.5-\alpha=0.75-\alpha=1$ (No porosity)

Figure 8: Sensitivity of the numerical solutions (root mean square errors) to the choice penalization permeability $\epsilon$ and porosity $\alpha$ coefficients in the upwelling test case with $h_{\min }=50 \mathrm{~m}$. 


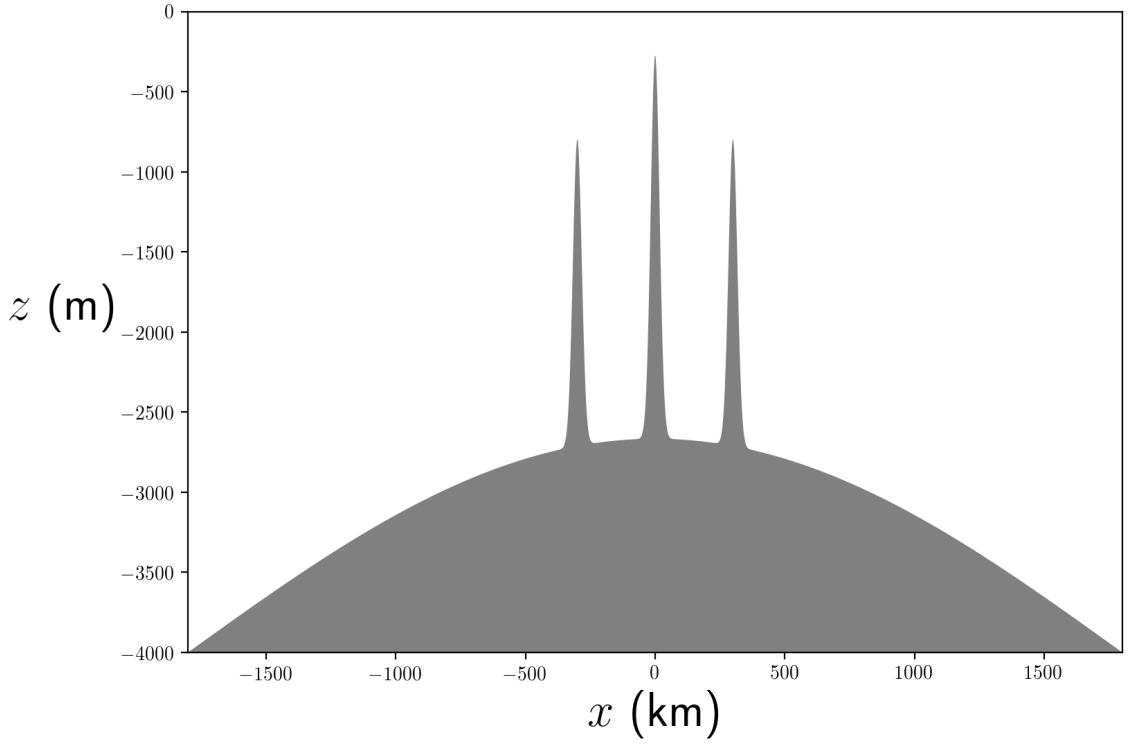

Figure 9: Idealized bathymetry for the internal tide test case.

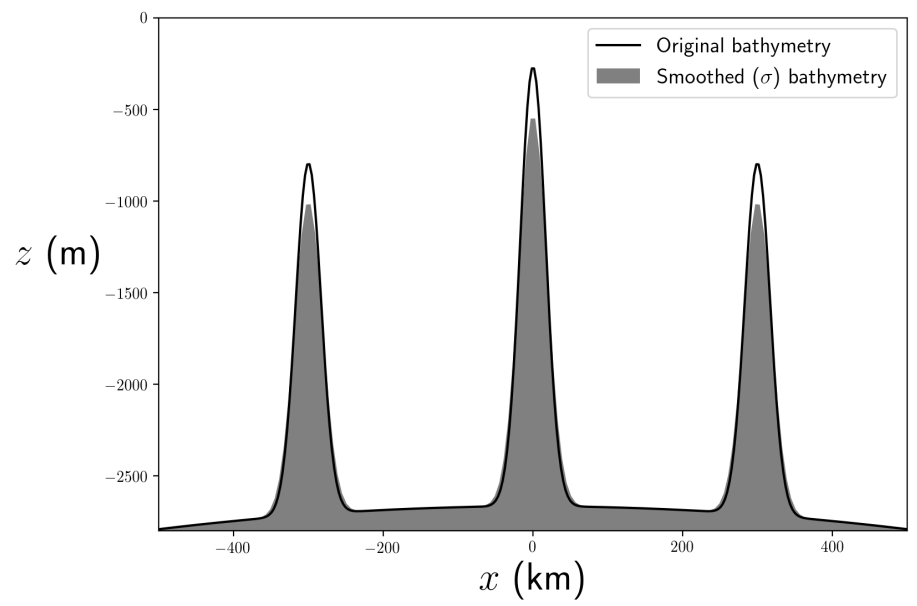

Figure 10: Reference and smoothed (coarse resolution) bathymetries for the internal tide test case.

The smoothed bathymetry satisfies the pressure gradient error criterion $r_{\max } \leq 0.2$ for $\sigma$-coordinates at $6 \mathrm{~km}$ horizontal resolution. 


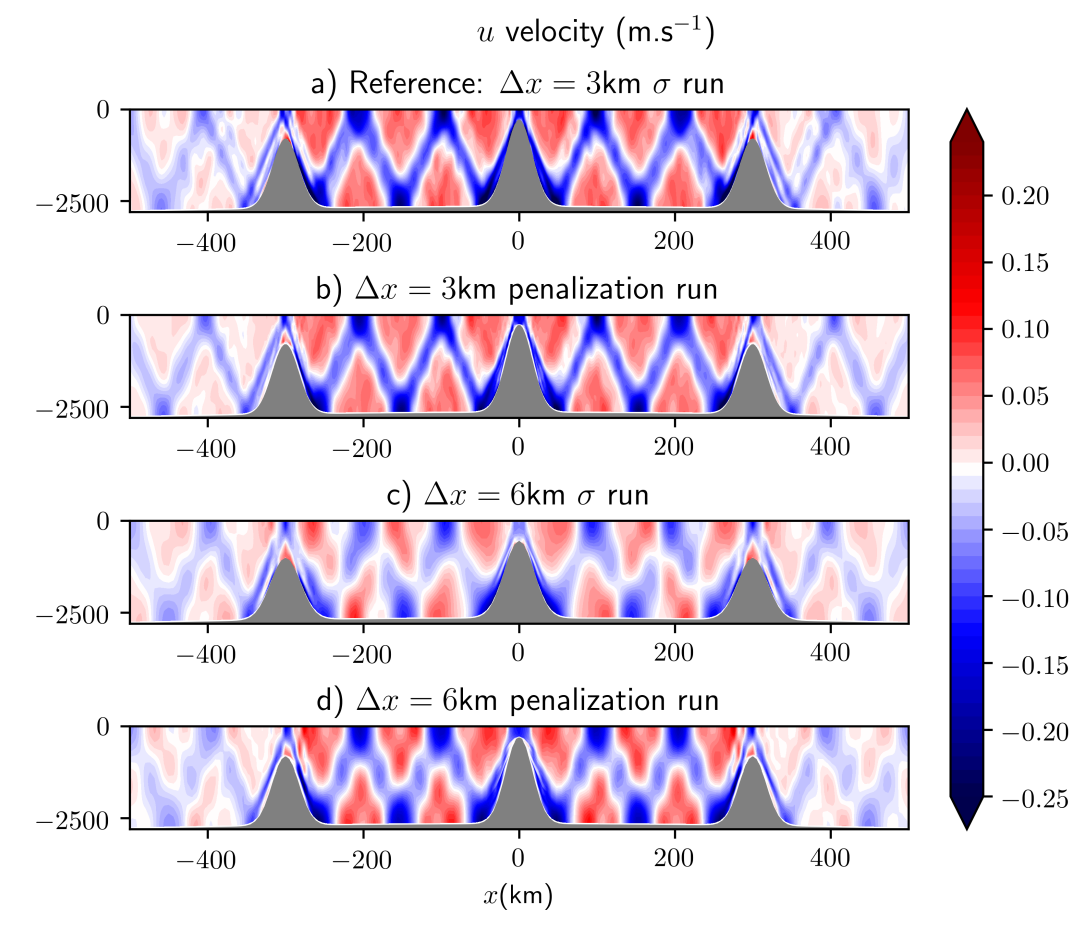

Figure 11: $u$ velocity. Instantaneous solutions of the internal tide test case after $12 M_{2}$ tidal cycles of integration. (a) The reference $\sigma$ coordinate run at $3 \mathrm{~km}$ resolution. (b) The penalized run at $3 \mathrm{~km}$ resolution. (c) The $\sigma$-coordinate run at $3 \mathrm{~km}$ resolution. (d) The penalized run at $6 \mathrm{~km}$ resolution. 